\title{
AEDES AEGYPTI AND DENGUE VIRUS INFECTIONS
}

\author{
By
}

\section{TOSSON A. MORSY}

\author{
Department of Parasitology, Faculty of Medicine, Ain Shams University, \\ Cairo 11566, Egypt (correspondence:morsyegypt2014@gmail.com)
}

\section{Abstract}

Aedes-borne diseases (Dengue, Chikungunya, Yellow Fever and Zika) are an emerging problem worldwide, escalating overall risk and burden of disease worldwide. Dengue viruses are members of the family Flaviviridae, genus flavivirus with four anti-genically related but distinct viruses; all are mosquito-borne human pathogens. The four dengue virus serotypes are associated with fever, rash, and the more severe forms, hemorrhagic fever and shock syndrome.

Susceptible humans become infected after being bitten by an infected female Aedes mosquito. Viremia in humans begins at the end of a four-six-day incubation period and persists until fever abates. An uninfected Aedes spp. may acquire the virus after feeding on a patient at the vermeil period. Infected mosquitoes carry the virus for their lifespan and pass the virus to its immature stages (Trans-ovarian transmission) and remain infective for humans,

Several factors have combined to produce epidemiological conditions in developing countries in the tropics and subtropics that favor viral transmission by the main mosquito vector, Aedes as the rapid population growth, rural-urban migration, inadequate basic urban infrastructure (eg. the unreliable water supply leading householders to store water in containers close to homes) and the increase in volume of solid waste, such as discarded plastic containers and other abandoned items which provide larval habitats in urban areas. Geographical expansion of the mosquito has been aided by the international commercial trade particularly in used car-tyres which easily accumulate rainwater. Increased air travel and the breakdown of vector control measures have also contributed greatly to the global burden of dengue and DH fevers.

Key words: Aedes aegypti, Dengue fever, mini-review.

\section{Introduction}

The viral etiology of dengue was established by the 1940s, and records of denguelike illness date back more than 200 years (Kroeger et al, 2004). Major changes in the epidemiology of dengue virus infections began after World War II and have continued to date. Besides, the major influence of the increased shipping and air-traffic globally, other major factors for the reemergence of dengue fever included the ecological and demographic changes in the tropical zones (Gubler, 2002). This gave current estimation of 50 to 100 million infections worldwide each year and over 2.5 billion individuals at risk for infection, the dengue viruses are now arguably the most important arthropod-borne viruses from a medical and public health perspective (Guzman et al, 2010).

Classification: Dengue viruses are members of the family Flaviviridae, genus flavivirus. The dengue virus complex comprises four antigenically related but distinct vi- ruses, designated dengue virus serotypes 1 through 4. All dengue viruses are mosquitoborne human pathogens that exclusively cause acute infection (Wilder-Smith and Schwartz, 2005).

Transmission: Both epidemic and endemic transmission of dengue viruses are maintained through a human-mosquito-human cycle involving mosquitoes of the genus $A e-$ des (Stegomyia). Transmission of dengue viruses between mosquitoes and nonhuman primates has been demonstrated to occur in Asia and Africa, but there is no evidence that such transmission is an important reservoir for transmission to humans (Wang et al, 2000)

Susceptible humans become infected after being bitten by an infected female Aedes mosquito. The viremia in humans begins towards the end of a four to six-day incubation period and persists until fever abates, which is typically three to seven days. An uninfected Aedes mosquito may acquire the 
virus after feeding on the subject during this vermeil period. The mosquito has an incubation period of 8 to 12 days before it is capable of transmitting the virus to susceptible people. Once infected, mosquitoes carry the virus for their lifespan and remain infective for humans (Vaughn et al, 1997).

Vectors: Aedes (Stegomyia) aegypti mosquitoes, the principal vector for the transmission of dengue virus, have many characteristics that make them ideal for dissemination of the virus (Halstead, 1984). A. aegypti typically breed in or close to houses, laying eggs in both man-made and natural water containers. The typical flight distance is relatively short. Ae. aegypti are daytime feeders that prefer to bite humans and are frequently unnoticed. They are easily interrupted in their feeding and move on to another host, frequently taking multiple blood meals in a single breeding cycle (Harrington et al, 2005). Thus, an infected A. aegypti may transmit dengue virus to several persons in a small area. For these reasons, family members who are at home during the day, typically women and young children, are thought to be at particularly high risk for infection (Scott et al, 2000).

A. aegypti are widely distributed in tropical and subtropical areas from latitude $45^{\circ}$ North to $35^{\circ}$ South. Efforts to control urban yellow fever in the Americas, which is also principally transmitted by $A$. aegypti, had greatly restricted the distribution of the mosquitoes in the Western hemisphere as of 1970 , but the mosquitoes have since reinfested nearly all of their former habitats (McBride et al, 1998).

A. albopictus mosquitoes are a competent vector for the transmission of dengue virus under both experimental and natural conditions but are less likely to do so since they do not bite humans as frequently as $A$. aegypti and more often breed in outdoor water containers. A. albopictus also are more tolerant of the cold and have a wider geographic distribution than A. aegypti (Gratz, 2004). However, they appear to be less efficient natural vectors for dengue virus; it has been rare to find either endemic transmission or large outbreaks in regions that have Ae. albopictus but not Ae. aegypti. Both Aedes species are also competent vectors for the chikungunya virus transmission, which has led to simultaneous outbreaks of both diseases in some areas (CDC, 1989). Other Aedes species were suspected of dengue virus transmission in isolated outbreaks in the $\mathrm{Pa}$ cific islands. But, these species played an insignificant role in the global of dengue virus transmission (Savage et al, 1998).

Patterns of transmission: Dengue virus transmission follows two general (but not mutually exclusive) patterns, with different implications for disease risk in both the local population and travelers.

Epidemic dengue: Epidemic dengue transmission occurs when the introduction of dengue virus into a region is an isolated event involving a single virus strain. If sufficiently large populations of susceptible hosts and mosquitoes are present, transmission of dengue is explosive, leading to a recognizable epidemic. The incidence among susceptible persons often reached 25 to $50 \%$ and could be higher. Herd immunity, changes in weather, and mosquito control efforts can all contribute to the termination of the epidemic (Caron, et al, 2012).

Transmission of dengue viruses prior to World War II almost exclusively followed this pattern. Seaports frequently were the point of initial introduction of dengue viruses, and these port cities then acted as distribution points to nearby inland areas (Gubler, 1997).

Epidemic activity is currently the predominant pattern of dengue virus transmission in smaller island nations, certain areas of South America and Africa, and in the areas of Asia where dengue virus transmission has recently reemerged. The incidence of dengue virus infections in these locations varies considerably from year to year. Intervals of several years or more usually pass between epidemics, allowing the number of susceptible indi- 
viduals to accumulate so that the next epidemic was perpetuated (Alvarez et al, 2006).

The implications of a predominantly epidemic pattern of dengue transmission are: 1The risk for acquisition of dengue virus for travelers is high during an epidemic but low at other times. However, outbreaks may be relatively difficult to predict. 2- Both adults and children are affected in the local population. 3- The frequency of dengue hemorrhagic fever (DHF) has usually been low during epidemics, with some significant exceptions. The viral serotype and strain and the interval since the previous epidemic seem to influence the risk for this manifestation of infection (Kouri et al, 1989).

Hyperendemic dengue: Hyperendemic transmission refers to the continuous circulation of multiple dengue virus serotypes in the same area. This requires the year-round presence of competent vector mosquitoes and either a large population base or steady movement of individuals into the area to maintain a pool of susceptible individuals. Hyperendemic circulation involves the occurrence of multiple epidemics in a smaller geographic scale e.g., village or school (Endy et al, 2002).

Seasonal variation in virus transmission is common. The incidence of infection also varies from year to year, with increased dengue transmission at intervals of three to four years, but this variation is not as dramatic as in areas where transmission predominantly follows the epidemic pattern. A recent mathematical analysis of data from Thailand suggested that these surges in dengue transmission originate in waves from major urban centers (Cummings et al, 2004). Areas with hyperendemic dengue virus transmission contribute the vast majority of cases of dengue virus infection globally. In some regions, $5 \& 10 \%$ of the susceptible population experiences dengue virus infection annually (Porter et al, 2005). Urban areas are particularly affected.

The implications of hyperendemic transmission of dengue virus are: 1- The overall risk for acquisition of dengue virus is higher for travelers than in areas that experience only epidemic transmission, but the seasonal variation in risk is somewhat predictable. 2The prevalence of antibody against dengue virus rises with age for the local population, and most adults are immune to infection. 3While children are more likely than adults to experience mild disease, hyperendemicity appears to be a major factor contributing to the occurrence of DHF. Dengue hemorrhagic fever is distinguished from dengue by the presence of increased vascular permeability, not by the hemorrhage (WHO, 1997). Patients with dengue may have severe hemorrhage without meeting WHO criteria for dengue hemorrhagic fever. Pathogenesis in these cases was derived from thrombocytopenia or a consumptive coagulopathy and not from the vascular leak syndrome of dengue hemorrhagic fever (Krishnamurti et al, 2001). Dengue hemorrhagic fever may of grades III \& IV or not grades I \& II include clinical shock, referred to as dengue shock syndrome. Dengue virus antigen was found in a variety of tissues, predominately liver and reticuloendothelial system (McBride and Bielefeldt-Ohmann, 2000). Viral replication occurs primarily in the macrophages, although dendritic cells (Langerhans cells) in the skin may be an early target of infection (Wu et al, 2000). As in yellow fever, focal central necrosis was found in the dead dengue patients' liver (Innis, 1995). Autopsies of patients who died of dengue haemorrhagic fever showed diffuse petechial haemorrhages in most organs and serous effusions of pericardial, peritoneal, and pleural spaces (Lum et al, 1996). Dengue virus (by isolation and RT-PCR) and IgM antibody were identified in cerebrospinal fluid, but the direct involvement of dengue virus in neuronal damage was controversial (Solomon et al, 2000). In the children, dengue fever gave atypical presentation like the splenomegaly, encephalopathy, bleeding manifestations and upper respiratory tract infection (Arshad et al, 2015). 
All four serotypes were associated with dengue hemorrhagic fever. Variations in the virus strains within and between the four serotypes may influence disease severity. Secondary infections (particularly serotype 2) are more likely to result in severe disease and dengue hemorrhagic fever (Thein et al, 1997). Theoretically, the antibody dependent enhancement, whereby cross reactive but non-neutralizing antibodies from a previous infection bound to the new serotype infection and facilitated virus entrance into cells causing higher peak viral titres (Sullivan, 2001). In primary and secondary infections, higher viral titres were associated with more severe disease (Vaughn et al, 2000). Higher titres might result in an amplified cascade of cytokines and complement activation causing endothelial dysfunction, platelet destruction (Lei et al, 2001), and consumption of coagulation factors, which result in plasma leakage and haemorrhagic manifestations (Kurane and Takasaki, 2001).

Increased vector density: In many tropical countries, seasonal increases in rainfall contribute to an increased density of mosquitoes. One factor that can be modified is the presence of open water storage containers in or near the home.

Shorter incubation period in the mosquito: The length of the incubation time in the mosquito, known as the extrinsic incubation period, is inversely associated with the ambient temperature. Warmer temperatures therefore increase the length of time that a mosquito remains infective.

Increased movement of mosquito vectors and viruses: Air, land, and water transportation of mosquitoes or vermeil humans facilitate the dissemination of dengue viruses.

Increased density of susceptible human hosts: Crowded conditions probably increase the potential for virus transmission. However, as the prevalence of prior infection increases, the fraction of the population that remains susceptible is reduced.

Increased duration and magnitude of viremia in humans: Attenuated virus strains pro- duced in the laboratory have been shown to produce low titers of virus in the blood, which are then not efficiently transmitted to mosquitoes (Schoepp et al, 1991). It is unclear whether natural strains of dengue virus can differ in the viremia titers they produce (Gubler et al, 1981).

Other routes of transmission: Given the high titers of infectious dengue virus found in blood and tissues during acute infection, it is not surprising that the potential exists for virus transmission by routes other than mosquito vectors:

Nosocomial transmission: At least four we11-documented cases of transmission of dengue virus in the health care setting via need le-stick and one case of transmission by nonpercutaneous exposure were reported (Chen and Wilson, 2004). Some blood donors, although asymptomatic, have viremia levels sufficient to transmit infection to recipients of the blood components. Nosocomial transmission cases in dengue-endemic regions (also likely to encompass majority of exposures) would usually be overlooked due to the inability to distinguish nosocomial transmission from mosquito transmission (Stramer et al, 2012). Needle-stich injury must be considered (Abdel Motagaly et al, 2017).

Vertical transmission: Vertical transmission of dengue has been reported in a few small case series (Sirinavin et al, 2004), based on these cases and the known pattern of viremia, this possibility should be considered in cases where illness in the mother occurs within the 10 days before delivery (including onset on the delivery day). Illness presented in these newborns up to 11 days (median 4 days) after birth. Although data are limited, pregnancy does not appear to increase the incidence or severity of dengue (Carroll et al, 2007). In a prospective study conducted in Kuala Lumpur of 2958 parturient, 2531 paired maternal-umbilical cord blood samples were tested for dengue-specific IgM to determine the prevalence of infection and the vertical transmission rate. Sixtythree women $(2.5 \%)$ had a positive IgM se- 
rology. Only one $(1.6 \%, 95 \%$ CI $0-9.5 \%)$ of the paired umbilical cord samples was seropositive for dengue. None of the maternal and fetal blood samples had evidence of viral RNA by PCR (Tan et al, 2008).

Factors contributing to the reemergence of dengue and dengue hemorrhagic fever: The worldwide incidence of dengue and DHF has been increasing in the past several decades, and the geographic distribution of these diseases has expanded. The emergence of DHF as a public health problem has largely been a result of human behaviors including: 1- Population growth. 2- Poorly planned urbanization, associated with overcrowding, poor water distribution, and poor sanitation. 3- Changing lifestyles, such as increased reliance on plastic containers and tires; standing water can easily collect within. 4- Modern transportation, with increased movement of viruses, mosquitoes, and susceptible humans. 5- Lack of effective mosquito control

The potential effects of global climate change would be a major source for future concern. Increased dengue virus transmission was associated with El Nino/Southern Oscillation events (Hales et al, 1996). Mathematical models predict that increased global temperatures would further expand $A$. aegypti and dengue virus range (Hales et al, 2002).

Distribution of $A$. aegypti and reported dengue activity: Currently A. aegypti and dengue viruses are endemic in every continent except Europe and Antarctica, although epidemic DHF occurs predominantly in Asia and the Americas. The risk for exposure to A. aegypti was often higher in urban areas. Many tourist facilities present a lower risk than local residential areas because of air conditioning, ground-skeeping, elevation, or combinations of these factors (Pinheiro and Corber, 1997).

The number of cases of dengue fever (DF) and DHF reported globally varied considerably from year to year, although the overall trend is one of increasing incidence. Global epidemics of disease have occurred at intervals of several years, most recently from
2001 to 2002. Several regions have reported increased dengue disease activity from 2007 through 2009. The reported dengue activity in specific regions is described further below. Most available information was gathered through passive surveillance activities and must be interpreted cautiously in light of underreporting of cases and lack of laboratory confirmation (WHO, 2012).

Generally, clinically diagnosed DF was widespread during the $18^{\text {th }}$ and $19^{\text {th }}$ centuries in North and South America, the Caribbean Basin, Asia, and Australia. In the Americas, this was largely due to the repeated introduction from Africa of A. aegypti (Reinert et al, 2008).

In Europe: In mainland France, where $A e$ des albopictus was present and disseminating since 2004, 2 episodes of autochthonous transmission occurred in 2010 and in 2013 during its activity period from May 1 to November 30 (Paty, 2014).

Southeast Asia and Western Pacific regions: Southeast Asia: A. aegypti are present throughout the region, extending to southern China and the south of the island of Taiwan, and all countries in the region are affected by dengue virus infections. Hyperendemic transmission of all four dengue serotypes, with cases of DHF, was present in Thailand, Vietnam, and Indonesia for over 40 years. Epidemic dengue reemerged in China during the 1980 s \& the 1990s after absence of several decades; associated with the first DHF occurrence in that country (Qiu et al, 1993) Nepal, the last country in the region to be affected, had the first cases detected in 2004. Dengue virus transmission occurred yearround, but typically reaches a seasonal peak which varies in timing from one country to another (for example, between June \& November in Thailand, but between January \& February in Indonesia). More than 200,000 cases of DHF were reported from the region annually from 2007 through 2009; Indonesia accounted for the majority of cases in each of these years.

South Asia: A. aegypti are present in most 
of the region. Hyperendemic transmission of all four dengue serotypes is present in Malaysia and the Philippines. Other islands experience frequent dengue outbreaks; high rates were reported from 14 island nations for 2009, including American Samoa, Cook Islands, French Polynesia, New Caledonia, and Tonga (Weaver and Reisen, 2010).

Western Pacific islands: A. aegypti are present in most of the region. Hyperendemic transmission of all four dengue serotypes is present in Malaysia and the Philippines. Other islands experience frequent dengue outbreaks; in the first half of 2009, over 1000 cases were reported from French Polynesia

Australia: Ae. aegypti are present in the northeastern corner of Australia. Dengue viruses are not endemic to the continent, but periodic introduction of dengue viruses from neighboring islands led to epidemics in urban areas of north Queensland during the 1990s and 2000s (WHO, 1998)

African and Eastern Mediterranean regions: A. aegypti are present in much of subSaharan Africa and the Middle East. Data are scant on dengue virus transmission. But, documented infections in visitors to the area indicated the ongoing dengue virus transmission (Sharp et al, 1995). Several outbreaks were reported from central and east Africa and the Middle East during the 1990s and the 2000s (Eisenhut et al, 1999).

Region of the Americas: In North America Ae. aegypti are present in most areas of Mexico and in the southeastern United States. Ae. albopictus is also present in these areas, but its range extends further north, nearly to the Great Lakes. Hyperendemic transmission of all four dengue virus serotypes is present throughout the range of $A e$. aegypti in Mexico. Dengue virus transmission is seasonal, with peak activity in late summer and fall. In 2011, over 67,000 cases of dengue infection were reported from Mexico, including 4000 cases of severe dengue and 36 deaths (Valerio et al, 2012).

Most dengue virus infections identified in the continental United States and all cases identified in Canada were acquired during travel abroad or to Puerto Rico or the United States Virgin Islands (CDC, 2013). Limited transmission of dengue virus (3 to 30 cases) within southern Texas was documented in 1980, 1986, 1995, 1997, \& 1998 (CDC, 1998) and an outbreak involving over 120 cases of locally acquired dengue infection occurred in Hawaii in 2001 (Effler et al, 2005). However, the CDC (2007) reported a case of DHF in a resident native to Texas who lived in a bordering area with Mexico. This prompted a serosurvey of 346 households in the immediate neighborhood, which demonstrated that 38 percent of the residents had IgG antibodies to dengue. Dengue fever was also reported in 28 residents of Key West, Florida who had not traveled abroad (CDC, 2010). A subsequent serosurvey of 240 participants living in Key West found that $5 \%$ had evidence of recent dengue infection. Local transmission of dengue virus was detected in south Florida, but still appears to be very limited in scope.

Central America: A. aegypti and hyperendemic transmission of all four dengue virus serotypes are present throughout the region. The region experienced a major outbreak in 2010; Honduras and Costa Rica reported among the highest numbers of cases of dengue $(67,000 \& 31,000$, respectively) and incidence rates (1016 \& 979 cases/100,000 populations) that year. However, all of these countries have had one or more years of heavy dengue activity during the past five years (CDC, 2005).

Caribbean: Ae aegypti is present throughout the region. Hyperendemic circulation of dengue virus serotypes $1,2, \& 4$ has been found on the larger islands (other than Cuba) for several decades and dengue virus serotype 3 has been present since 1998. In Puerto Rico, peak dengue virus transmission usually occurs between October and December; over 21,000 cases of dengue virus infection were reported there in 2010. The Dominican Republic (11,000 cases), French 
Guiana (4000 cases), Guadeloupe $(41,000$ cases), Martinique (37,000 cases), and St. Martin (2400 cases) all reported major outbreaks in 2010. Other islands experienced periodic dengue epidemics (CDC, 2005).

South America: A. aegypti is present in every South American country except Chile. Hyper-endemic circulation of all four dengue virus serotypes has been present in the north of the continent since the reintroduction of dengue virus serotype 3 was detected in Brazil and Venezuela during 2000. Brazil, Venezuela, and Colombia have reported the largest number of dengue cases. Low-level, year-round transmission has been observed, but most cases follow an epidemic pattern; in Brazil, peak dengue transmission occurred between February and May (Siqueira et al, 2005).

Brazil experienced a major outbreak in 2010 , concentrated in the state of Rio de Janeiro, and with over 1,000,000 cases nationwide, including over 16,000 cases of severe dengue. Colombia (150,000 cases) and Venezuela (120,000 cases) also reported major dengue outbreaks in 2010. Comparison of disease incidence in Asia and Latin America showed a distinct difference in age distribution of DF and DHF. In Asia, hospitalizations principally involve children, whereas in the Americas, they tend to involve a greater proportion of adults (Halstead, 2006). The reasons for this apparent difference were not adequately defined. However, an epidemic in the State of Rio de Janeiro revealed that the incidence of DHF in children was significantly higher than in previous epidemics in Brazil (Cavalcanti et al, 2011).

Diagnosis: diagnosis of dengue fever is based on the detection of NS1 Ag, antibodies (IgM \& IgG) or reverse transcription (RT)-PCR (virus isolation is used less often). For early diagnosis (onset $<5$ days), detection of NS1 Ag might be used, but its moderate sensitivity required the presence of both NS1 Ag and IgM to ensure diagnosis (Chaterji et al, 2011). IgM were positive 4 to
5 days after disease onset for up to 3 to 6 months. IgG appeared approximately 710 days after onset and were detectable thereafter for life. RT-PCR detection of viral RNA proved a very reliable technique for patients presenting within 5 to 7 days of the onset of symptoms, but this method is more expensive, non-standardized, and only a few centers in France use it routinely (Osorio et $a l, 2010)$. Consequently, serological tests are commonly used to establish or confirm a diagnosis of dengue. False-positive results include cross-reactive flavivirus-specific antibodies, nonspecific binding of antibodies secreted in the course of various infections such as mononucleosis or hepatitis, and rheumatoid factor. Wichmann et al. (2006) diagnosed 127 dengue fever cases by single positive rapid test. Each case was further investigated by several IgM- \& IgG-ELISAs, immunofluorescence assays, and RT-RT/PCR assays, leukopenia gave $42.5 \%$ false-positives and thrombocytopenia gave $6.1 \%$ falsepositives. So, positive rapid test results must be confirmed by laboratory-based ELISA or virus PCR detection for a dengue fever reliable diagnosis (Dechamps et al, 2009). Travellers might be infected with dengue virus because transmission is maintained even between epidemics; malaria must be ruled out in those returning from an endemic area with symptoms (Magill, 1998). In Australia and Germany up to $8 \%$ of travellers returning with febrile illnesses were found to have dengue (O'Brien et al, 2001). Because the incubation period can vary from 3 to 14 days; typically between $5 \& 7$ days and viraemia could persist up to 12 days; typically 4 to 5 days (Rigau-Perez et al, 1998), dengue can be ruled out if symptoms begin more than 2 to 3 weeks after the patient has left an endemic area or if the fever lasts more than two weeks. But, dengue hemorrhagic fever and dengue shock syndrome are rare in travellers; but those with a history of dengue must be advised to protect themselves well from mosquitoes when in endemic areas (Allwinn et al, 2008). Pancer et 
al. (2014) reported that dengue proved to be a serious condition with the presence of $\operatorname{IgG}$ antibodies directed against antigens of particular serotypes, associated with primary infection caused by different serotype or transferred from infected mother to her child. For adequate dengue laboratory diagnosis, a set of various diagnostic methods must be done. Within the family Flaviviridae, cross-reactivity may lead to occurrence of false-positive results. In Poland, differential diagnosis with different Flavivirus species is of special importance as it is an endemic area for tick-borne encephalitis (TBE). Thus, data regarding history of patient's immunization against TBE or yellow fever should be also taken into consideration as important in interpretation of results of serological examination. But, without specific symptoms, febrile illness diagnosis in returning travelers was challenging. Pull et al. (2012) reported that cosmopolitan infections such as measles must be considered, even more as its incidence was increasing in several parts of the world. Kutsuna et al. (2015) reported that clinical manifestations, simple laboratory test results, and regions of travel are helpful to distinguish between dengue, malaria, and enteric fever in febrile returning travelers with non-specific symptoms.

In the Middle East and North Africa: Ae. aegypti originated in Africa where its ancestral form was a zoophilic treehole mosquito named Ae. aegypti formosus (Brown et al, 2014). Many countries in the EMR (which includes Afghanistan, Bahrain, Djibouti, Egypt, Iraq, Iran, Jordan, Kuwait, Lebanon, Libya, Morocco, Oman, Pakistan, Palestine, Qatar, Saudi Arabia, Somalia, Sudan, Syria, Tunisia, United Arab Emirates and Yemen) are particularly vulnerable to communicable disease epidemics, because they are experiencing numerous environmental and social stresses, including armed conflicts, water scarcity, food insecurity, rapid population growth and urbanization (Kraemer et al, 2015). But, the host availability was the most important predictor in case of Ae. albo-pictus.
Dengue fever has a historic presence in the Middle East and North Africa (MENA), with outbreaks of dengue and dengue-like disease reported across much of the Eastern Mediterranean region in the $19^{\text {th }}$ and early $20^{\text {th }}$ centuries (Garabedian et al, 1971).

Nowadays, dengue may be resurging in the MENA (WHO, 2014) with outbreaks of unprecedented or previously unrecognized magnitude occurring in Pakistan (Rai, 2011) and the Arabian Peninsula (Arya and Agarwal, 2014) and an outbreak in Egypt that occurred following a decades-long absence of reported cases from that country (WHO, 2015). Still, despite increasing global concern about the threat of Aedes-transmitted arboviruses, the epidemiology of dengue in the MENA region was largely uncharacterized (Humphrey et al, 2016).

Ducheyne et al. (2018) stated that in the Middle East and North Africa, the epidemiology of dengue remains poorly characterized despite increasing reports of outbreaks and transmission in new areas. They added that there was wide spread distribution and the abundance of Ae. aegypti and the reported cases of dengue, chikungunya and yellow fever. Tran Minh et al. (2016) assumed that the potential risk of disease outbreaks was high in at least eight of the EMR countries: Djibouti, Egypt, Oman, Pakistan, Saudi Arabia, Somalia, Sudan and Yemen. The invasive vector Ae. albopictus spread in some countries, such as Lebanon (2012) and Moracco (2016) but the available occurrence database was not updated. They conducted that a systematic review of studies reporting human prevalence, incidence, and infection rat- es in the virus' main mosquito vectors, Ae. aegypti and Ae. albopictus. They identified, the Red Sea sub-region and Pakistan reported the highest seroprevalence estimates for dengue fever.

In Egypt, Kirkpatrick (1925) reported Ae. aegypti. Gad (1963) identified Ae. aegypti, Ae. caspius and Ae. detritus. Holstein (1967) reported complete eradication of Ae. aegypti from Egypt. Mikhail et al. (2009) reported 
Ae. caspius and Ae. detritus.

Burdino et al. (2011) in North West Italy identified two imported cases of DENV infections from South Egypt in patients travelling together, confirming the importance of returning travelers as sentinels of a rapidly changing epidemiology in specific geographic areas. They concluded there must a careful evaluation and follow-up of febrile patients returning from Dengue endemic countries. Heikal et al. (2011) reported the re-emergence in Aswan and approach of $\mathrm{Ae}$ des aegypti, the vector of the Dengue hemorrhagic fever, Yellow fever and Chikungunya fever, which are encountered in Africa, needs to alert for this public health threat. The presence of dengue and dengue hemorrhagic fever in Saudi Arabia is another issue. El-Bahnasawy et al. (2011) dengue (DF) and dengue hemorrhagic fevers (DHF) are present in urban and suburban areas in the Americas, South-East Asia, the Eastern Mediterranean and the Western Pacific, but dengue fever is present mainly in the rural areas of Africa. They concluded that the presence of Ae. aegypti and endemicity of DF \& DHF in the neighboring regional countries must be in mind of the public health authorities.

Saleh (2012) reported immature and matures stages of the tiger mosquito, Ae. aegypti in surveying Aswan Governorate. Shoukry et al. (2012) reported the re-emergence Ae. aegypti in the Egyptian southern border (Aswan Governorate) and added that now in Toshka District is an integration mark.

WHO (2015) in October reported a dengue outbreak with at least 253 cases was reported from Assiut Governorate. Abozeid et al. (2018) reported the first detection of Ae. aegypti from an ongoing dengue outbreak in Red Sea Governorate, with more than 680 cases to date. They sampled Hurghada and Al-Quseir and found Cx. pipiens, and Ae. aegypti. They suggested that Ae. aegypti might originate from Saudi Arabia, having crossed the Red Sea on ships, or from Sudan via ground traffic, and the urgent surveillance of Ae. aegypti and other vector species, such as Ae. albopictus, which is currently spreading worldwide.

Prevention: Prevention of dengue as other insect-borne infectious diseases must focus on the vector. Ae. aegypti is difficult to control owing to its intimacy with man. The following were some selected control measures

Rodríguez et al. (2002) in Cuba found that pyrethroid resistance was associated with a glutathione-S-transferase mechanism but not esterases. The cross-resistance to deltamethrin from temephos selection could limit the use of both insecticides for vector control. Shaalan et al. (2005) in Egypt studied sublethal concentrations of fenitrothion, lambda-cyhalothrin \& Callitris glaucophylla $(\mathrm{Cu}-$ pressaceae) extract to characterize their effects on the Ae. aegypti development. They recommended the botanical for field application either in combination with synthetic or natural insecticides or alone. El-Hela et al. (2013) in Egypt conducted targeting estimation of the relative levels of total phenolic content, antioxidant potential and larvicidal activity of 110 selected medicinal plants. They concluded that their polyphenols constitute a distinct group of natural compounds with wide range of physiological activities as antioxidant, immunestimulant, antitumor and antiparasitic. Coelho (2012) in Brazil reported that the vector control activities recommended by both the National Program for Dengue Control and programs from other countries was the home visit to carry out the inspection of deposits placed in and outdoors, Ae. aegypti potential breeding sites. Gamal (2012) in Saudi Arabia used Gambusia holbrooki versus Bacillus thuringiensis israelensis (B.t.i.) to control Ae. aegypti larvae in domestic co-ntainers. The infestation rate was significantly higher $(\mathrm{P}<$ $0.001)$ in the B.t.i group. In deposits where the fish remained, efficacy was $85 \%$ better than B.t.i. He concluded that the permanence of fish was higher in concrete tanks (48.5\%) located outside the house $(47.5 \%)$ and at ground level (53.3\%). Jacups et al. (2013) in Australia studied the efficacy and longevity 
of triflurumon Starycide ${ }^{\circledR}$ (I.G.R.) under field conditions in tropical against Aedes in doses ( $0.48 \& 0.96 \mathrm{ppm})$, applied to water receptacles with delayed flooding by zero, two, and four weeks. They found that triflurumon gave excellent efficacy and longevity in artificial containers. If done under standard conditions, reapplication was not needed for up to 22 weeks. Mackay et al. (2013) in Puerto Rico developed an autocidal gravid ovitrap (AGO) as a simple, low-cost device for surveillance and control of Ae. aegypti without the use of pesticides that does not require servicing for an extended period of time. They concluded that the AGO-B could be used to efficiently attract and capture gravid Ae. aegypti females for more than 8 weeks without the need for trap maintenance. Winskill et al. (2014) in the United Kingdom reported that release of male $A e$. aegypti carrying a dominant lethal (RIDL) could be employed to control. To maximize the RIDL potential control, optimum release strategies for trans-genic mosquitoes were needed. They concluded that maximizing the public health benefits of RIDL-based vector control would involve optimizing all stages of the control program. They added that to achieve long-term suppression of a wild population combined releases of adults and pupae were recommended. Quimbayo et al. (2014) in Spain evaluated 18 different ovitraps of combined insecticides (deltamethrin and permethrin), oviposition substrates (towel, dacron and flannel), and attractant infusion (10 \& 20\% hay infusion, and dechlorinated water).They found the ovitrap combining deltamethrin, towel as oviposition substrate, and $10 \%$ hay infusion as attractant was the most efficient control. Afify et al. (2014) in Germany used a modified Y-tube olfactometer to test the effect of some chemical compounds on Ae. aegypti flying towards skin odor (human fingers), and their effect on oviposition choice in a cage assay. They found that EA \& DEET are good repellent, but MDA is repellent only in a hostseeking context, and BA is deterrent only in an oviposition context.

Nagpal et al. (2016) in India hypothesized that whether continuous entomological surveillance and simultaneous appropriate interventions in key containers during nontransmission months would have any impact on breeding of Aedes and dengue cases during the transmission months. The surveillance and intervention measured undertaken during non-transmission months assessed by entomological indicators namely container index (CI), house index (HI), pupal index (PI) and breteau index (BI). They concluded that proper intervention in non-transmission season reduced vector density and subsequently dengue cases in transmission season.

\section{Conclusion}

Aedes aegypti are daytime feeders that frequently unnoticed bites man. They are characterized by interrupted feeding and move on to another host, frequently taking multiple blood meals in a single breeding cycle. Thus, it can transmit dengue virus to several persons within a small geographic area.

Epidemic dengue transmission occurs by introduction of dengue virus into a region as an isolated event involving a single virus strain. If sufficiently large persons of susceptible hosts and vector are present, transmission is explosive, leading to a recognizable epidemic. The herd immunity, changes in weather, and mosquito control efforts can all contribute to the epidemic termination. Dengue virus transmission is enhanced by multiple factors, as higher vector density, its greater movement, and increased density of susceptible humans.

Hyperendemic transmission is the continuous circulation of multiple dengue virus serotypes in the same area. This requires the year-round presence of competent vector \& an ongoing presence of susceptible individuals. Reinfection with dengue different type may lead to a serious, life-threatening condition. Half of patients (mainly children) developed symptoms of dengue fever, dengue haemorrhagic fever or dengue shock syndrome. Fatality is high in severe dengue. Unless 
Aedes vectors can be effectively controlled or a cost effective vaccine developed, dengue can be expected to continue to escalate.

\section{References}

Abdel-Motagaly, AME, Ibrahim, AMA, Morsy, TA, 2017: An intervention program on blood protozoa acquired by needle stick injury and infection control. J. Egypt. Soc. Parasitol. 47, 2: 309-22.

Abozeid, S, Elsayed, AK, Schaffner, F, Samy, AM, 2018: Re-emergence of Aedes aegypti in Egypt. Lancet Infect. Dis. 18, 2:142-3

Afify, A, Horlacher, B, Roller, J, Galizia, CG, 2014: Different repellents for Aedes aegypti against blood-feeding and oviposition. PLoS One 9, 7:e103765.

Allwinn, R, Hofknecht, N, Doerr, HW, 2008: Dengue in travellers is still underestimated. Intervirology 51, 2:96-100.

Alvarez, M, Rodriguez-Roche, R, Bernardo, L, et al, 2006: Dengue hemorrhagic fever caused by sequential dengue 1-3 virus infections over a long time interval: Havana epidemic, 20012002. Am. J. Trop. Med. Hyg. 75, 6:1113-7.

Arshad, K, Sheikh, S, Naqvi, SU, Sarwar, I, Javaid, S, et al, 2015: Frequency of splenomegaly in dengue fever in children. J. Ayub Med. Coll. Abbottabad. 27, 2:356-9.

Arya, SC, Agarwal, N, 2014: Apropos: An update on the incidence of dengue gaining strength in Saudi Arabia and current control approaches for its vector mosquito. Parasites Vectors 7, 1: 233-8

Bennouna, A, Balenghien, T, El Rhaffouli, H, Schaffner, F, Garros, C, et al, 2016: First record of Stegomyia albopicta (= Aedes albopictus) in Morocco: a major threat to public health in North Africa? Med. Vet. Entomol. 31:102-6. doi: 10.1111/mve.12194.

Brown, JE, McBride, CS, Johnson, P, Ritchie, S, Paupy, C, et al, 2011: Worldwide patterns of genetic differentiation imply multiple 'domestications' of Aedes aegypti, a major vector of human diseases. Proceed. Biol. Sci.; the Roy. Soc. 278:2446-54.

Burdino, E, Milia, MG, Sergi, G, Gregori, G, Allice, T, et al, 2011: Diagnosis of dengue fever in North West Italy in travelers from endemic areas: a retrospective study J. Clin. Virol. 51, 4: 259-63.

Caron, M, Paupy, C, Grard, G, et al, 2012: Recent introduction and rapid dissemination of
Chikungunya virus and Dengue virus serotype 2 associated with human and mosquito coinfections in Gabon, central Africa. Clin. Infect. Dis. 55:e45.

Carroll, ID, Toovey, S, Van Gompel, A, 2007: Dengue fever and pregnancy- a review and comment. Travel Med. Infect. Dis.5:183-8.

Cavalcanti, LP, D. Vilar, D, Souza-Santos, R, Teixeira, MG, 2011: Change in age pattern of persons with dengue, Northeastern Brazil, Emerg. Infect. Dis. 17, 1:132-4.

CDC, 1989: Update: Aedes albopictus infestation--United States, Mexico. MMWR Morb. Mortal. Wkly. Rep. 38:440, 445.

CDC, 1998: Imported dengue--United States, 1996. MMWR Morb. Mortal. Wkly. Rep. 47: 544-8.

CDC, 2005: Travel-associated dengue infections--United States, 2001-2004. MMWR Morb. Mortal. Wkly. Rep. 54:556-8.

CDC, 2007: Dengue hemorrhagic fever--U.S.Mexico border, 2005. MMWR Morb. Mortal. Wkly. Rep. 56:785-9.

CDC, 2010: Locally acquired Dengue--Key West, Florida, 2009-2010. MMWR Morb. Mortal. Wkly. Rep. 59:577-9.

CDC, 2013: Notes from the field: school reporting of a dengue outbreak st. Croix, U.S. Virgin islands, 2012. MMWR Morb. Mortal. Wkly. Rep. 62:172.

Chaterji, S, Allen, JC, Jr, Chow, A, et al, 2011: Evaluation of the NS1 rapid test and the WHO dengue classification schemes for use as bedside diagnosis of acute dengue fever in adults. Am. J. Trop. Med. Hyg. 84:224-8

Chen, LH, Wilson, ME, 2004: Transmission of dengue virus without a mosquito vector: nosocomial mucocutaneous transmission and other routes of transmission. Clin. Infect. Dis. 39:e56.

Coelho, GE, 2012: Challenges in the control of Aedes aegypti. Rev. Inst. Med. Trop. Sao Paulo. 54, 18:S13-4.

Cummings, DA, Irizarry, RA, Huang, NE, et al, 2004: Travelling waves in the occurrence of dengue haemorrhagic fever in Thailand. Nature 427:344.

Dechamps, M, Jouret, F, Zech, F, Lambert, M, 2009: Fausse dengue, rougeole vraie, à propos d'un cas. Louvain Méd. 128:145-8.

Ducheyne, ELS, Tran Minh, NN, Haddad, N, Bryssinckx, W, Buliva, E, et al, 2018: Current and future distribution of Aedes aegypti and Ae- 
des albopictus (Diptera: Culicidae) in WHO Eastern Mediterranean Region. Int. J. Hlth. Geogr.

17:4. doi: 10.1186/s12942-018-0125-0

Effler, PV, Pang, L, Kitsutani, P, et al, 2005: Dengue fever, Hawaii, 2001-2002. Emerg. Infect. Dis. 11:742-50.

Eisenhut, M, Schwarz, TF, Hegenscheid, B, 1999: Seroprevalence of dengue, chikungunya and Sindbis virus infections in German aid workers. Infection 27:82.

El-Bahnasawy, MM, Khalil, HH, Morsy, AT, Morsy, TA, 2011: Threat of dengue fever and dengue haemorrhagic fever to Egypt from travelers. J. Egypt. Soc. Parasitol. 41, 2:289-306.

El-Hela, AA, Abdel-Hady, NM, Dawoud, GT, Hamed, AM, Morsy, TA, 2013: Phenolic content, antioxidant potential and Aedes aegyptii ecological friend larvicidal activity of some selected Egyptian plants. J. Egypt. Soc. Parasitol. 43, 1:215-34.

Endy, TP, Nisalak, A, Chunsuttiwat, S, et al, 2002: Spatial and temporal circulation of dengue virus serotypes: a prospective study of primary school children in Kamphaeng Phet, Thailand. Am. J. Epidemiol.156:52-5.

Gad, AM, 1963: Insects of Medical Importance. Res. Inst. Med. Entomol. Ministry of Health, Dokki, Giza, Egypt.

Gamal, ZA, 2012: Effectiveness of Gambusia holbrooki fish in domestic water containers and controlling Aedes aegypti larvae (Linnaeus, 1762) in southwest Saudi Arabia (Jeddah). J. Egypt. Soc. Parasitol. 42, 1:1-10.

Gratz, NG, 2004: Critical review of the vector status of Aedes albopictus. Med. Vet. Entomol. 18:215-9.

Gubler, DJ, 1997: Dengue and dengue hemorrhagic fever: Its history and resurgence as a global public health problem. In: Dengue and Dengue Hemorrhagic Fever, Gubler, DJ, Kuno, G (Eds), CAB International, Wallingford.

Gubler, DJ, 2002: The global emergence/resurgence of arboviral diseases as public health problems. Arch. Med. Res. 33, 4:330-42.

Gubler, DJ, Suharyono, W, Lubis, I, et al, 1981: Epidemic dengue 3 in central Java, associated with low viremia in man. Am. J. Trop. Med. Hyg. 30:1094-102.

Guzman, MG, Halstead, SB, Artsob, H, et al, 2010: Dengue: A continuing global threat. Nat. Rev. Microbiol. 8:S7-12.

Haddad, N, Mousson, L, Vazeille, M, Chamat, $\mathbf{S}$, Tayeh, J, et al, 2012: Aedes albopictus in
Lebanon, a potential risk of arboviruses outbreak. BMC Infect. Dis. 12:300. doi: 10.1186/ 1471-2334-12-300.

Hales, S, de Wet, N, Maindonald, J, Woodward, A, 2002: Potential effect of population and climate changes on global distribution of dengue fever: an empirical model. Lancet 360:830.

Hales, S, Weinstein, P, Woodward, A, 1996: Dengue fever epidemics in the South Pacific: driven by El Niño Southern Oscillation? Lancet 348:1664.

Halstead, SB, 1984: Selective primary health care: Strategies for control of disease in the developing world. XI. Dengue. Rev. Infect. Dis. 6: 251-8.

Halstead, SB, 2006: Dengue in the Americas and Southeast Asia: do they differ? Revista Panamericana de Salud Publica, 20, 6:407-15.

Harrington, LC, Scott, TW, Lerdthusnee, K, et al, 2005: Dispersal of the dengue vector Aedes aegypti within and between rural communities. Am. J. Trop. Med. Hyg. 72:209-12.

Heikal, OM, El-Bahnasawy, MM, Morsy, AT, Khalil, HH, 2011: Aedes aegypti re-emerging in Egypt: a review and what should be done? J. Egypt. Soc. Parasitol. 41, 3:801-14.

Holstein, M. 1967: Dynamics of Aedes aegypti: Distribution, density and seasonal prevalence in the Mediterranean Area. Bull. Org. Mon. Santre, WHO 36: 541-3.

Innis, BL, 1995: Dengue and dengue hemorrhagic fever. In: Kass Handbook of Infectious Diseases: Exotic Virus Infections. Porterfield JS, editor, London: Chapman and Hall Medical.

Jacups, SP, Paton, CJ, Ritchie, SA, 2013: Residual and pre-treatment application of starycide insect growth regulator (triflumuron) to control Aedes aegypti in containers. Pest Manag Sci. 70, 4:572-5

Kirkpatrick, TW, 1925: Mosquitoes of Egypt. The Egyptian Government Press.

Kouri, GP, Guzmán, MG, Bravo, JR, Triana, C, 1989: Dengue haemorrhagic fever/dengue shock syndrome: lessons from the Cuban epidemic, 1981. Bull. WHO 67:375.

Kraemer, MUG, Sinka, ME, Duda, KA, Mylne, A, Shearer, FM, et al, 2015: The global compendium of Aedes aegypti and Ae. albopictus occurrence. Sci, Data. 2:150035. doi: 10.1038/ sdata.2015.35

Krishnamurti, C, Kalayanarooj, S, Cutting, MA, Peat, RA, Rothwell, SW, et al, 2001: Mechanisms of hemorrhage in dengue without circ- 
ulatory collapse. Am. J. Trop. Hyg. 65:840-7.

Kroeger, A, Nathan, M, Hombach, J, 2004: WHO/TDR Reference Group on Dengue. Dengue Nat. Rev. Microbiol. 2:360-4.

Kurane, I, Takasaki, T, 2001: Dengue fever and dengue haemorrhagic fever: challenges of controlling an enemy still at large. Rev. Med. Virol. 11:301-11.

Kutsuna, S, Hayakawa, K, Kato, Y, Fujiya, Y, Mawatari, M, et al, 2015: Comparison of clinical characteristics and laboratory findings of malaria, dengue, and enteric fever in returning travelers: 8-year experience at a referral center in Tokyo, Japan. J. Infect. Chemother. 21, 4:272-6.

Lei, HY, Yeh, TM, Liu, HS, Lin, YS, Chen, S $\mathbf{H}$, et al, 2001: Immunopathogenesis of dengue virus infection. J. Biomed. Sci. 8:377-88.

Lum, LC, Lam, SK, Choy, YS, George, R, Harun, F, 1996: Dengue encephalitis: A true entity? Am. J. Trop. Med. Hyg. 54:256-9.

Mackay, AJ, Amador, M, Barrera, R., 2013: An improved autocidal gravid ovitrap for the control and surveillance of Aedes aegypti. Parasit Vectors 6, 1:225.

Magill, AJ, 1998: Fever in the returned traveler. Infect. Dis. Clin. North Am. 12:445-69.

McBride, WJ, Bielefeldt-Ohmann, H, 2000: Dengue viral infections; pathogenesis and epidemiology. Microbes Infect. 2:1041-50.

McBride, WJ, Mullner, H, LaBrooy, JT, Wronski, I, 1998: The 1993 dengue 2 epidemic in North Queensland: a serosurvey and comparison of hemagglutination inhibition with an ELISA. Am. J. Trop. Med. Hyg. 59:457-9.

Mikhail, MW, Al-Bursheed, KhM, Abd ElHalim, AS, Morsy, TA, 2009: Studies on mosquito borne diseases in Egypt and Qatar. J. Egypt. Soc. Parasitol. 39, 3:745-56.

Nagpal, BN, Gupta, SK, Shamim, A, Vikram, K, Srivastava, A, et al, 2016: Control of Aedes aegypti breeding: A novel intervention for prevention and control of dengue in an endemic zone of Delhi, India. PLoS One 11, 12:e01667 68. doi: 10.1371/journal.pone.0166768.

O'Brien, D, Tobin, S, Brown, GV, Torresi, J, 2001: Fever in returned travelers: review of hospital admissions for a 3-year period. Clin. Infect. Dis. 33:603-9.

Osorio, L, Ramirez, M, Bonelo, A, et al, 2010: Comparison of the diagnostic accuracy of commercial NS1-based diagnostic tests for early dengue infection. Virol. J. 7:361-8.

Pancer, K, Szkoda, MT, Gut, W, 2014: Impo- rted cases of dengue in Poland and their diagnosis. Przegl. Epidemiol. 68, 4:651-5.

Paty, MC, 2014: Dengue fever in mainland France. Arch. Pediatr. 21, 11:1274-8.

Pinheiro, FP, Corber, SJ, 1997: Global situation of dengue and dengue haemorrhagic fever, and its emergence in the Americas. World Health Stat Q. 50:161.

Porter, KR, Beckett, CG, Kosasih, H, et al, 2005: Epidemiology of dengue and dengue hemorrhagic fever in a cohort of adults living in Bandung, West Java, Indonesia. Am. J. Trop. Med. Hyg. 72:60-6.

Pull, L, Brichler, S, Bouchaud, O, Siriez, JY, 2012: Differential diagnosis of dengue fever: Beware of measles. J. Travel Med. 19, 4:268-71.

Qiu, FX, Gubler, DJ, Liu, JC, Chen, QQ, 1993: Dengue in China: a clinical review. Bull WHO 71:349.

Quimbayo, M, Rúa-Uribe, G, Parra-Henao, G, Torre C, 2014: Evaluation of lethal ovitraps as a strategy for Aedes aegypti control. Biomed. 34, 3:473-82.

Rai, MA, 2011: Epidemic: Control of dengue fever in Pakistan. Nature 479, 7371:41.

Reinert, JF, Harbach, RE, Kitching, IJ, 2008: Phylogeny and classification of Ochlerotatus and allied taxa (Diptera: Culicidae: Aedini) based on morphological data from all life stages. Zool. J. Linnean Soc. 153, 1:29-114.

Rigau-Perez, JG, Clark, GG, Gubler, DJ, Reiter, P, Sanders, EJ, et al, 1998: Dengue and dengue haemorrhagic fever. Lancet 352:971-7.

Rodríguez, MM, Bisset, J, Ruiz, M, Soca,A, 2002: Cross-resistance to pyrethroid and organophosphorus insecticides induced by selection with temephos in Aedes aegypti (Diptera: Culicidae) from Cuba. J. Med. Entomol. 39, 6:882-8.

Saleh, NM, 2012: Aedes mosquito in Aswan Governorate, Egypt. J. Egypt. Soc. Parasitol. 42, 1:233-8.

Savage, HM, Fritz, CL, Rutstein, D, et al, 1998: Epidemic of dengue-4 virus in Yap State, Federated States of Micronesia, and implication of Aedes hensilli as an epidemic vector. Am. J. Trop. Med. Hyg. 58:51922.

Schoepp, RJ, Beaty, BJ, Eckels, KH, 1991: Infection of Aedes albopictus and Aedes aegypti mosquitoes with dengue parent and progeny candidate vaccine viruses: a possible marker of human attenuation. Am. J. Trop. Med. Hyg. 45: 202-8.

Scott, TW, Amerasinghe, PH, Morrison, AC, 
et al, 2000: Longitudinal studies of Aedes aegypti (Diptera: Culicidae) in Thailand and Puerto Rico: blood feeding frequency. J. Med. Entomol. 37:89-93.

Shaalan, EA, Canyon, DV, Younes, MW, Abdel-Wahab, H, Mansour, AH, 2005: Effects of sub-lethal concentrations of synthetic insecticides and Callitris glaucophylla extracts on the development of Aedes aegypti. J. Vector Ecol. 30, 2:295-8.

Sharp, TW, Wallace, MR, Hayes, CG, et al, 1995: Dengue fever in U.S. troops during Operation Restore Hope, Somalia, 1992-1993. Am. J. Trop. Med. Hyg. 53:89-92.

Shoukry, NM, Elwan, MA, Morsy, TA, 2012: Aedes aegypti (Linnaeus) re-emerging in southern Egypt. J. Egypt. Soc. Parasitol. 42, 1:41-50.

Siqueira, JB, Jr, Martelli, CM, Coelho, GE, et al, 2005: Dengue and dengue hemorrhagic fever, Brazil, 1981-2002. Emerg. Infect. Dis.11:48-52.

Sirinavin, S, Nuntnarumit, P, Supapannachart, S, et al, 2004: Vertical dengue infection: Case reports and review. Pediatr. Infect. Dis. J. 23:1042.

Solomon, T, Dung, NM, Vaughn, DW, Kneen, R, Thao, LT, et al, 2000: Neurological manifestations of dengue infection. Lancet 355:1053-9.

Stramer, SL, Linnen, JM, Carrick, JM, et al, 2012: Dengue viremia in blood donors identified by RNA and detection of dengue transfusion transmission during the 2007 dengue outbreak in Puerto Rico. Transfusion 52:1657.

Sullivan, NJ, 2001: Antibody-mediated enhancement of viral disease. Curr. Trop. Microbiol. Immunol. 260:145=69.

Tan, PC, Rajasingam, G, Devi, S, Omar, SZ, 2008: Dengue infection in pregnancy: prevalence, vertical transmission, and pregnancy outcome. Obstet. Gynecol.111:1111-9.

Thein, S, Aung, MM, Shwe, TN, Aye, M, Zaw, A, et al, 1997: Risk factors in dengue shock syndrome. Am. J. Trop. Med. Hyg. 56:56672.

Tran Minh N, Huda, Q, Asghar, H, Samhouri, D, Abubakar, A, Barwa, C, et al, 2016: Zika virus: no cases in the Eastern Mediterranean Region but concerns remain. East. Mediterr. Hlth. J. 22:350-5. doi: 10.26719/2016.22.5.350.

Valerio, L, Facchinelli, L, Ramsey, JM, Bond, JG, Scott, TW, 2012: Dispersal of male Aedes aegypti in a coastal village in southern Mexico. Am. J. Trop. Med. Hyg. 86, 4:665-76
Vaughn, DW, Green, S, Kalayanarooj, S, et $a l$, 1997: Dengue in the early febrile phase: Viremia and antibody responses. J. Infect. Dis. 176: 322-6.

Vaughn, DW, Green, S, Kalayanarooj, S, Innis, BL, Nimmannitya, S, et al, 2000: Dengue viremia titer, antibody response pattern, and virus serotype correlate with disease severity. J. Infect. Dis. 181:2-9.

Wang, E, Ni, H, Xu, R, et al, 2000: Evolutionary relationships of endemic/epidemic and sylvatic dengue viruses. J. Virol. 74:3227-32.

Weaver, SC, Reisen, WK, 2010: Present and future arboviral threats. Antiviral Res. 5, 2:32845.

WHO, 1997: Dengue haemorrhagic fever: diagnosis, treatment, prevention and control, $2^{\text {nd }}$ edn. Geneva.

WHO, 1998: Dengue in the WHO Western Pacific Region. Wkly. Epidemiol. Rec. 73:273, Geneva, Switzerland.

WHO, 2012: Report on Global Surveillance of Epidemic-Prone Infectious Diseases. www. who. int/csr/resources/publications/dengue/CSR/ISR_ 2000_1/en.

WHO, 2014: Growing threat of viral haemorrhagic fevers in the Eastern Mediterranean Region: a call for action Regional Office for the Eastern Mediterranean. Cairo, Egypt. Available at http://applications.emro.who.int/docs/em_rc544 _en.pdf?ua $=1$.

WHO, 2015: Dengue fever-Egypt http://www. who.int/csr/don/12-november-2015-dengue/en.

Wichmann, O, Stark, K, Shu, PY, et al, 2006: Clinical features and pitfalls in the laboratory diagnosis of dengue in travellers. BMC Infect. Dis. 6:120-8

Wilder-Smith, A, Schwartz, E, 2005: Dengue in travelers. N. Engl. J. Med. 353:924-8.

Wu, SJ, Grouard-Vogel, G, Sun, W, Mascola, JR, Brachtel, E, et al, 2000: Human skin Langer-hans cells are targets of dengue virus infection. Nat. Med. 6:816-20.

Winskill, P, Harris, AF, Morgan, SA, Stevenson, J, Raduan, N, et al, 2014: Genetic control of Aedes aegypti: data-driven modelling to assess the effect of releasing different life stages and the potential for long-term suppression. Parasit. Vectors 7:68. doi: 10.1186/1756-3305-7-68. 